\title{
Some Guidelines on the Engineering Applications of Elastic-Plastic Fracture Mechanics
}

\section{F, J. WITT}

Westinghouse Electric Corporation, Power Systems Division, P.O. Box 2728, Pittsburgh, Pennsylvania 15230-2728, USA

\section{ABSTRACT}

Eiastic-plastic $J$-integral fracture mechanics analyses are performed rather routinely. It is first shown that the accuracy of the results depends significantly on the accuracy with which the material true-stress truestrain curve is fit. Using exactly the same stress strain curve approximation, two independent finite element analyses of a circumferential flaw in a pipe are shown to yield similar results. Plastic zone size corrections to linear elastic methods are reasonably accurate up to near the engineering yield stress but become unacceptably non-conservative above this value.

sponsorship of EPRI are shown to produce accurate results as the elastic plastic regime is entered if the Ramberg-0sgood fit of the stress strain curve is good in that region. Further into the elastic-plastic region reasonably good. A Ramberg-Osgood fit to the mid-strain range (2 to $5 \%)$ is shown to be unacceptably conservative. Some guidel ine for obtaining

acceptable approximations of finite element analyses in the elastic plastic region are given.

\section{KEYWORDS}

Cracked pipes, elastic-plastic, finite element, fracture mechanics, J-integral, Ramberg-Osgood, stress-strain.

\section{INTRODUCTION}

Elastic-plastic fracture mechanics analyses are performed rather routinely to assess the fracture potential of structures. One of the most commonly used approaches involves the J-integral methodology. The analytical too preferred is perhaps that of using finite element modelling. Such an
approach however may be quite costly, and simplified less expensive methods are often applied instead. 
Three fracture assessment methods - finite element, plastic-zone size correction and finite element interpolation - are addressed in this paper. Rather simple structures, large pipes with through-wall circumferential flaws subjected to axial bencing moments, are considered. Analyses are

\section{COMPARISON OF FINITE ELEMENT RESULTS}

It is generally assumed that finite elements analyses yield the most accurate state-of-the-art solution for elastic-plastic fracture behavior and serve as benchmarks for evaluations of other procedures. As a first pproach finite element analyses were made of a 34 inch outside diameter (0.D.) pipe 25 inches thick, using two widely accepted computer codes, MARC (Marcal, 1969) and ADINA (Bathe, 1978) (modified for J-integral alculations). In the analysis using MARC the path integral was used to abtain $J$ for a given crack size and load whereas in the modified ADINA analysis the virtual crack extension method (Yang and Palusamy, 1983) was used. The pipe material was stainless steel. A generic lower bound true-stress true-strain curve (henceforth referred to as stress strain curve) typical of this material at $600^{\circ} \mathrm{F}$ was applied.

Two representations of the stress stain curve were taken as shown in Fig. 1. One representation was a five-point approximation of the curve and the other was a bilinear representation as seen. MARC and ADINA as modified were run using the five point approximation while only ADINA as modified was function of the applied moment. The crack length subtended an angle of $27^{\circ}$.

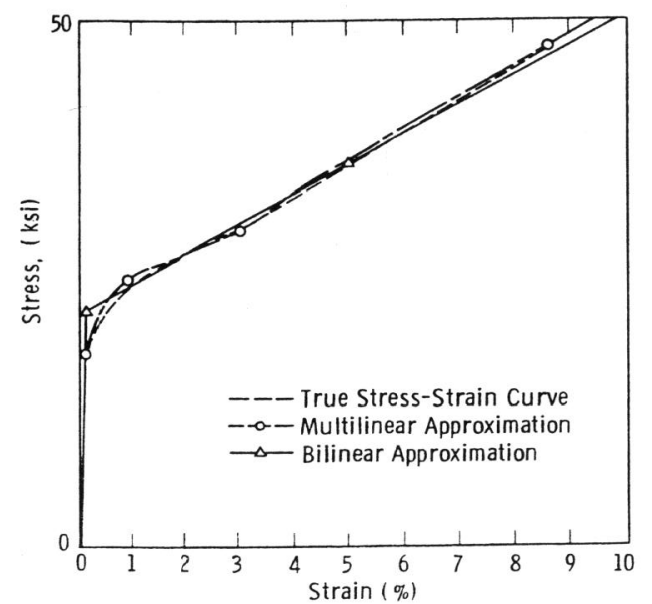

Fig. 1. Stress Strain Curve and Its Bilinear and Multilinar Approximations Used in Analyzing Multilinear Approximatio

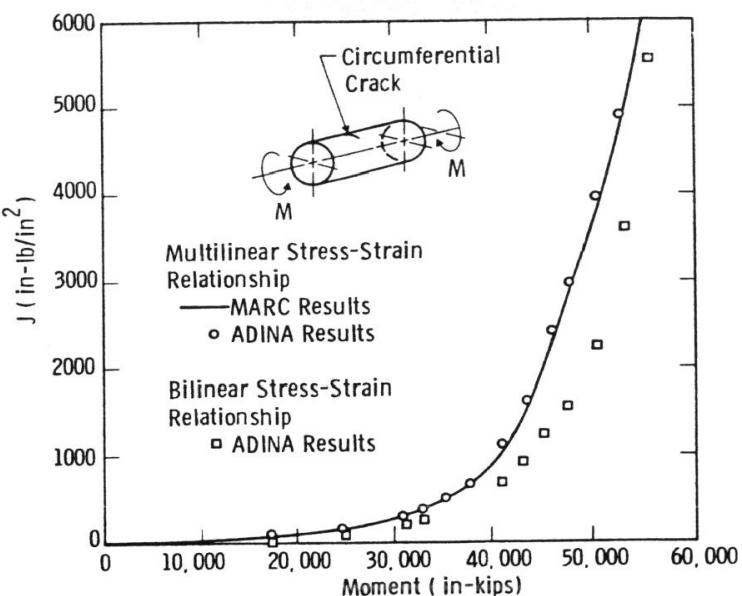

Fig. 2. Comparison of the Finite Element $\mathrm{J}$ Results for the 34 Inch O.D. Pipe Using MARC and ADINA.

There are two significant observations from Fig. 2. First, using the same tress strain curve almost identical results were obtained using two different finite esults using the bilinear representation compare very unfavorably in a non-conservative manner with the other results in the range of $\mathrm{J}$ from $500 \mathrm{in}^{-1 \mathrm{~b} / \mathrm{in}^{2}}$ to 5000 In-1b/in which spans the usual range of interest for stainless steel.

\section{PLASTIC ZONE SIZE CORRECTION METHOD}

One of the earlier approaches of addressing elastic-plastic behavior was to incorporate a plastic zone size correction into a linear elastic solution. for circumferential. flaw in pipes one such linear elastic solution for applied bending moments is

$$
K=\sigma_{b} \sqrt{\pi a} F(\alpha)
$$

where $F(\alpha)$ is the stress intensity calibration factor for bending, a is the half angle length, $\alpha$ is the half-crack angle, and $\sigma_{p}$ is the remote fiber stress due to pure bending (Tada, 1983). Values or $F(\alpha)$ are given by Tada (1983). The effect of yielding near the crack tip can be addressed by incorporating the plastic zone size correction developed by Irwin (1960), Into equation (1) by replacing a by the effective half-crack length, a eff defined by

$$
a_{e f f}=a+k^{2} / 2 \pi \sigma_{y}^{2}
$$


where the term added to a is the plastic zone size correction and $\sigma$ is size for specific corrected $\mathrm{K}$ is $\mathrm{i}$ applications abound but all have the same character. The correcting the halfagain to obtain the final $K$. $J$ is found

$$
J=k^{2} / E
$$

where $E$ is Young's modulus.

This method was used to analyze the problem solved by the finite element method in the previous section. A comparison of solutions is given in Fig. 3. It is noted that the comparison is very favorable up to near wher the bending stress is equal to the yield stress. At this point the where zone size is slightly over one-half the half crack length. The plastictic size is usually required to be small, theoretically in . The plastic zone for quite a large size.

Results similar to those in Fig. 3 have been found for tensile loads and tensile and bending loads conbined. The specific definition of the plastic conservatism, one gross results somewhat but in general accuracy and conservatism, one gross yielding occurs, are lost. Calculationally, acceptable ander consideration is flaws, could further ron plastic zone size, such as for part through

\section{A FINITE ELEMENT INTERPOLATION METHOD}

As noted previously, finite element elastic-plastic J-integral solutions are validation state-of-the-art and as such tend to serve as benchmarks for developed under the approaches. A rather unique and popular approach is on and is presented in handices of be referred to as the handbook procedure.

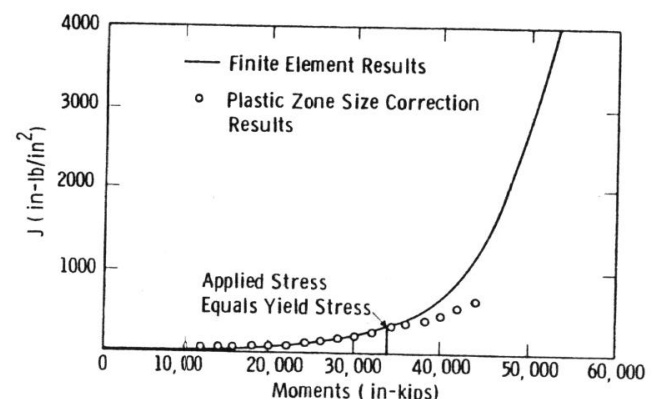

Fig. 3. Comparison of $J$ Results Using Plastic Zone Size Corrections and the Finite Element Results for the 34 Inch O.D. Pipe.
The handbook procedure is in fact an interpolation scheme based on finite lement results and would be expected to yield very accurate results if only interpolation were involved. However to provide a handbook methodology som croction handook (the

$$
\frac{\varepsilon}{\varepsilon_{0}}=\sigma / \sigma_{0}+\alpha\left(\sigma / \sigma_{0}\right)^{N}
$$

where $\sigma$ and $\varepsilon$ denote stress and strain, respectively. $\alpha$ and $N$ are the Ramberg-Osgood coefficients. Subscript 0 indicates the stress and strain at yield. Rigorously speaking, the yield stress (i.e., the 0.2 percent offset yield) has little relevance in finite-element elastic-plastic analyses. Th vield stress for purposes of this discussion is taken as the proportional imit defined as the point of deviation from linearity of the stress strain curve.

Equation (4) represents the stress-strain behavior of the material of interest here (i.e., stainless steel) only to an approximation. As seen later, the method of approximating the stress-strain behavior can have an inpact on J-integral results every bit as significant as changing flaw size, loading or geometry. Frequently, the key to performing an accurate or reasonably accurate analysis is the use of the proper $\alpha$ and $N$. This is

Thus the problem is not with the accuracy of the handbook procedure itself but with the Ramberg-Osgood coefficients. The applicability of the

hamberg-Osgood relationship to the stress-strain behavior of stainless stee has been evaluated by Landes and McCabe (1986) and it is concluded that a reasonably good fit cannot be obtained over the full strain range. This is especially true if the restriction on $\mathrm{N}$ discussed later is enforced.

As a rationale for approaching the problem of determining the Ramberg-Osgood coefficients, there are certain facts which are helpful. First and foremost, it is generally known that when the nominal stress produces requirement regime is entered is interest as strain hardening maintain accuracy as more plasticity occurs, the stress-strain, that to interest must continue to be reasonably described by the Ramberg-Osgof fit. It is also reasonable to judge that if the stress-strain relationship is not reasonably well fit as early plasticity occurs then the J-integral results may not be accurate in that stress region and will be questionable ht higher plastic regions until such a high strain is obtained for which oorlier effects are minimized.

The impact of the stress strain curve on the $\mathrm{J}$ results are readily seen in 199. 2. Thus the approximation of the stress strain curve is of great

Once having an acceptable stress strain curve available for an analysis, the handbook procedure requires an additional step - that of determining adequate values of the Ramberg-Osgood coefficients. In general, the (4) to 7 or less for the configuration under consideration. 
To illustrate the method for representing the stress strain curve for applying the handbook procedure, a 16 inch 0.0 . pipe, 1.6 inches thick was chosen from several examples available. The materia given in Fig. 4. the representative lower bound stress strain curverified for two circumFinite element analyses were run using ADINA as modified for two circumferential flaw sizes, one having an ang appoximation of the stress-strain finite element analyses used a ten point approximation of the stras as the curve which well defined the early stages of strain hardening corresponding strain latter stages. A point consisting will be called a couple. The propted in Fig. 5 and compared with the couples. The ten couples ar the same nine strain-hardening couples as

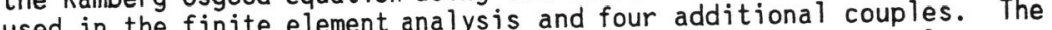
used in the fire couples were so proportional Fig. 5 the stress level of such a fit couple is plotted along with the strain obtained by the fit. The Ramberg-Osgood curve is also given in

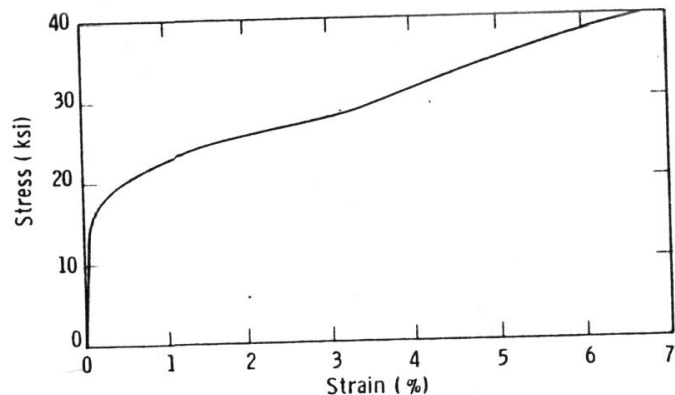

Fig. 4. Stress Strain Curve Used in Analyzing the 16 Inch O.D. Pipe.

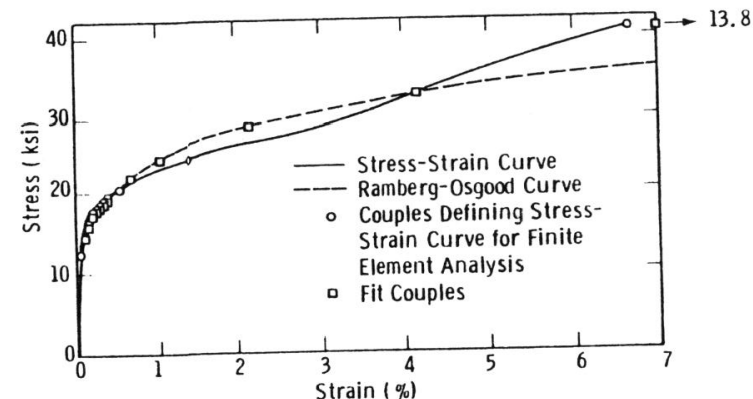

Fig. 5. Finite Element Approximation and Ramberg-Osgood Approx Strain Curve Used in Analyzing the 16 Inch OD Pipe 14g. 5. The Ramberg-Osgood curve starts out slightly below the stresstrain curve, crosses between 19000 and 19500 psi, ranges somewhat above it to near 32000 psi then drops significantly below it as the stress

ncreases. It is worth noting that at 40,000 psi the Ramberg-Osgood strain 5 and $\alpha$ was near 0.6 . Interestingly, if a set of data describing a stress strain curve is fit by
equation $(4), N$ is independent of the selected yield stress. However, if and $\sigma$ are two different selected values of yield stress, the crresponding $\alpha^{\prime} s$ are related by

$$
\alpha_{1}=\alpha_{0}\left(\sigma_{1} / \sigma_{0}\right)^{\mathrm{N}-1}
$$

while generally, the proportional limit is recommended in this paper for use as the yield stress in fitting a stress strain curve, using the usually more available engineering yield stress does not impact the $J$ result too adversely. For the handbook analyses considered in this paper using the engineering yield stress (about 60 percent greater than the proportional imit) as $\sigma$ produced $J$ values around 10 percent lower than that

obtained using the proportional limit as o in the stress range between the proportional limit and the engineering yield stress. As the applied stress increases the difference becomess considerably less approaching 1 tress approaching 150 percent of the engineering yield stress.

inth finite element and handbook $J$ results were obtained for axial moment the stress strain curve as shown in

1i. 5. The results are compared for the larger flaw size in Fig. 6 and for

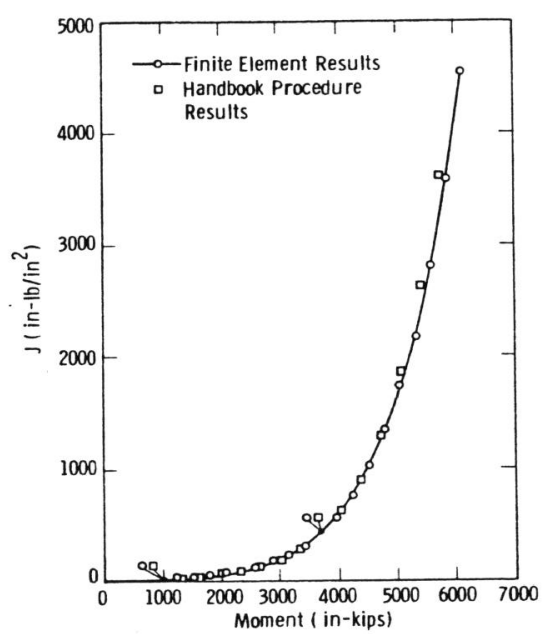

Fig. 6. Comparison of Handbook Procedure J Results with Those of the Finite Element Analysis for the 60 Degree Circumferential Flaw in the 16 Inch O.D. Pipe 
the smaller flaw size in Fig. 7. Excellent agreement is noted in Fig. 6 up to a moment of about 5000 in-kips which corresponds to a stress of around 21 ksi. It is noted in Fig. 5 , that the Ramberg-0sgood curve agrees well with the stress strain curve up to this level. The point of deviation of the results in Fig. 6 is somewhat surprising since the Ramberg-Osgood fit intersects and falls above the actual stress strain curve near the $21 \mathrm{ks}$ level. This does not detract from the excellent to conservative comparison, however, and indicates the somewhat compensating effect of underestimating and overestimating, within limits, the stress strain curve of interest.

The comparison of results for the smaller flaw is excellent over the whole loading regime as noted in Fig. 7. In particular the small flaw analysis appears to give a somewhat better comparison than the large flaw analysis.

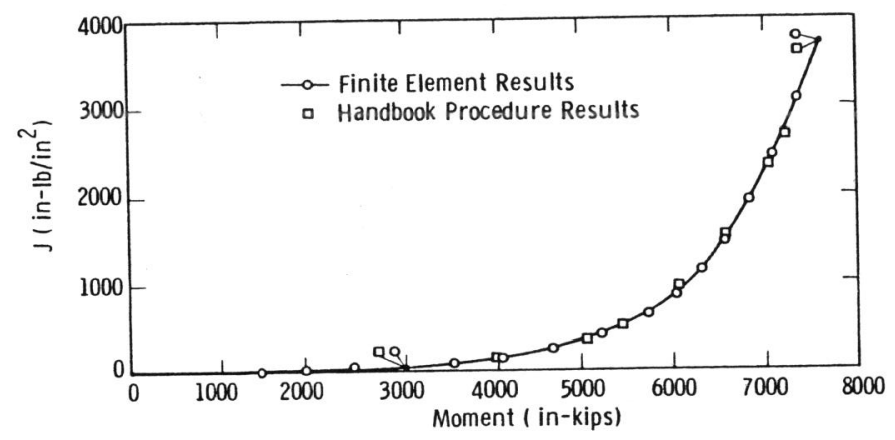

Fig. 7. Comparison of Handbook Procedure J Results with Those of the Finite Element Analysis for the 66 Degree Circumferential Flaw in the 16 Inch 0.D. Pipe

An analysis of the 16 in 0.D. cylinder using the handbook procedure was made Anased on a Ramberg-0sgood fit of the stress strain curve of Fig. 4 in the based on a Ramberg-Osgood fit of the stre fit compared with the stress strain mid strain range (2 to 5 percent). The falls well below the stress strain curve is given in ig. curve of interest in the early stramber that for a stresults from the handbook two greate for the larger flaw are compared with the corresponding finite procedure for the li Fis 9 . The handbook procedure results would ordinarily be considered as far to conservative for engineering applications. This is true for a loading stress level in the 2 to 5 percent strain range true en foress level for the finite element results presented goes up to around $26 \mathrm{ksi}$ (i.e. slightly over 2 percent strain).

The behavior presented in this section is typical of that for other loading and flaw configurations.

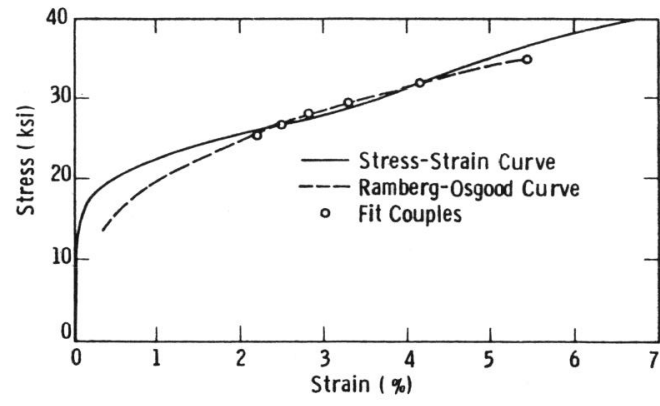

Fig. 8. Comparison of the Mid-Range Strain (2 to $5 \%$ ) Fit Ramberg-Osgood Curve with the Stress Strain Curve Used in the Finite Element Analysis of the 16 Inch O.D. Pipe

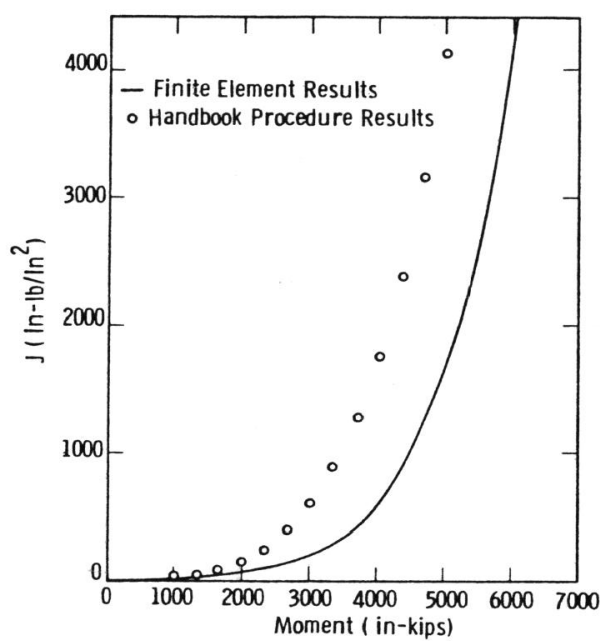

Fig. 9. Comparison of $\mathrm{J}$ Results Using the Handbook Procedure having a Mid-Range Strain Fit Ramberg-Osgood Curve With Those of the Finite Element Analysis for the $60^{\circ}$ Circumferential Flaw in the 16 Inch O.D. Pipe 


\section{DISCUSSION AND CONCLUSIONS}

a fircumferential flaws in a pipe are Finite benchmarked one against the us ang the same stress-strain representation. Thellent agreement was stress-strain representation yielded significantly The use of a differhich emphasizes the importance of the elastic-plastic different results which emphasizes the impore gross section yielding occurs. stress strain relationship in analyses wher crack tip An evaluation of plastic-zone corrections to account for crack tip near plasticity was shown to be reasonably acceptablic zone sizes are calculated. gross section yielding even though large platical considerations, Restrictions on plastic zane of applicability appreciably.

Various analyses using the handbook approach are compared with finite

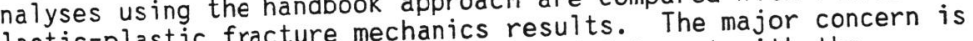
the stress strain curve of interest with the

thaterg-0sood expression for stress and strain. To obtain good comparison with the finite element results in the region of gross section yielding, good $f$ it of the Ramberg-Osgood equation in this region is requi of equation 4 as the proportional data produced 9 values about $10 \%$ low in this region. For higher loads the percentage decreased significantly. With in the restrictions of the handbook procedure the complete stress strain diagram cannot be adequately fit for the mater a l of interest in thise (i.e., stainless steel). Very good comparisons of handbook prob high loads results with finite element results were found when the stress strain curve was well fit by the Righer strains. With thes as gross yielding occurred and reasonably fither to agree very well with restrictions, the handbook results were show side of conservatism. the finite element results or to err on the side of conservatism.

Fitting the Ramberg-Osgood expression to the mid-strain range (2 percent to Wotentially unacceptably conservative even 5 percent strain) was seen to be success has been obtained at fitting at high load levels. Al though som stress strain curve for higher load Ramberg-Osgood expressions to the stres stra is judged that for those situations (not discussec in this paper), it be tolerated, finite element situations, where undue conate methods) may be the only recourse.

analyses (or equally accurate methods) may be the oniy recourse.

The results presented in this paper are representative of a larger analysis base and the conclusions and observations are based on the results presented, strengthened by adnless steel however.

Ductile tearing has not been addressed in this evaluation. If significant Ductile tearing has not seen then such tearing must be taken into account. tearing th Judge curve is known is discussed by simple procedu

SOME GUIDELINES

Desed represtive results presented in this paper the Based on the selected representative resultess steel components are judged following guidel astic plastic finite element fracture mechanics analyses should depict accurately the stress strain curve of the material of interest.

atic zone size corrections to curnear elculationally, but accurate up to near the

wen the Ramberg-Osgood expression cannot be made to fit accurately the the Ram stress strain curve with the restriction on $\mathrm{N}$ (e.g., $\mathrm{N}<7$ for the further mitigating the fit. the handbok procedure to accurately duplicate finite element results, the handbook procedure to accurately duply be fit by the Ramberg-Osgood the stress of interest.

of many applications the handbook procedure produces accurate or reasonably conservative results if both a good Ramberg-Osgood fit is obtained in the initial yielding region and a reasonably good fit to higher stress levels representative of the loading condition is obtained.

itting the Ramberg-Osgood equation to the mid strain range (2 to $5 \%$ ) is expected to produce results which may be restrictively conservative. te element analyses (on other equaliy accurate methods) appear to be the only recourse if accurate results are required for very high stres levels.

\section{REFERENCES}

Harcal, P.V. (1969). Finite Element Analysis of Combined Problems of , P.V. (1969). Finite Elenent Analysis of Conbined Prown University, 1969.

1969.j. (1978). A Finite Element Program for Automatic Dynamic (1) of Technology.

. C Y for a Pressurized Pipe Under Bending, Journal of Pressure Vessel Technology, 105, 16-22.

ad Factors and the Crack Opening Area of a Circumferential and a

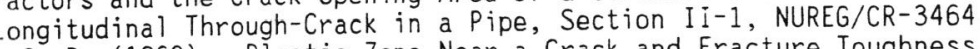

. Proceedings of the 7 th Sagamore Conference, IV-63.

Wuar, V., German, M. D., and Shih, C. P. (1981). An Engineering Approach for Elastic-Plastic Fracture Analys is, EPRI

amberg, W. and Osgood, W. R. (1943). Description of Stress-Strain Curves by Three Parameters, NACA TN 902.

by Three Parameters, NACA Toughness of Austenitic Stainless De F. J. (1987). The Shadowing Method of Elastic-Plastic Fracture

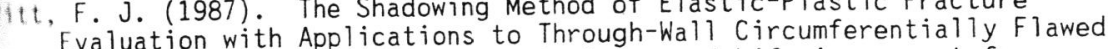
Pipes. In. Advances in Piping Analyses and Life Assessment for Pressure Vessels and Piping (Change et al, eds.), PVP 129, American Society of Mechanical Engineers, 55-60. 